\title{
Caracterização de dosímetros semicondutores para dosimetria in Vivo na técnica de TBI
} Characterization of Dosimeters Semiconductor for in vivo dosimetry in the technique of TBI Caroline Castilhano Sampaio ${ }^{1}$, Caroline Z. S. Emiliozzi ${ }^{2}$, Laura Furnari ${ }^{2}$

\author{
${ }^{1}$ Faculdade de Medicina FMUSP, Universidade de São Paulo, São Paulo, BR \\ ${ }^{2}$ Departamento de Radiologia e Oncologia, Hospital das Clinicas HCFMUSP, Faculdade de Medicina, \\ Universidade de São Paulo, São Paulo, BR
}

\section{Resumo}

A irradiação de corpo inteiro (TBI) é considerada uma técnica de alta a complexidade em radioterapia, já que exige campos alargados. Os esquemas de cálculo para fazer a determinação exata da distribuição da dose no TBI são complexos e variações na posição do paciente alteram dramaticamente as distribuições de dose. Sendo assim, é desejável ter uma técnica de medição in vivo disponível. Os diodos semicondutores são robustos, relativamente baratos e fornecem leituras on-line para inferência imediata de dose, o que é uma grande vantagem em comparação com a leitura do um dosímetro termoluminescente (TLD) que necessita de certo intervalo de tempo após a irradiação para sua análise. O objetivo deste trabalho é a caracterização e validação do diodo semicondutor (T600010L PTW) para utilização em medidas in vivo, e em tempo real, da dose liberada em um tratamento de irradiação de corpo inteiro. Os dosímetros devem ser caracterizados e calibrados para sua utilização na técnica em questão. Sendo assim foi feito o estudo da reprodutibilidade, da linearidade e da dependência da resposta dos diodos com a taxa de dose, com a distância fonte-detector (SSD) e com o ângulo de incidência do feixe de radiação. A calibração de cinco diodos semicondutores (T600010L PTW) foi realizada por intercomparação com uma câmara de ionização Farmer e sua verificação foi feita com a simulação de um tratamento em um phantom antropomórfico RANDO®. Uma vez que o dosímetro mostrou-se adequado e com variações menores que $1 \%$, estabeleceram-se limites de ação para dosimetria in vivo de acordo com a região a ser tratada e levando em conta fatores de incertezas que não são intrínsecos ao detector. O valor máximo encontrado para o limite de ação foi de 9,6\% na região do joelho.

Palavras-chave: dosimetria in vivo; dosímetro semicondutor; diodo; radioterapia; TBI.

\section{Abstract}

The total body irradiation technique (TBI) is considered a highly complex technique in radiotherapy since it requires broad fields. Calculation schemes to make the exact determination of dose distribution in the TBI are complex and variations in the position of the patient dramatically alter the dose distributions. Thus, it is desirable to have an in vivo measurement technique available. Semiconductor diodes are robust, relatively inexpensive, and provide online readings for immediate dose inference, which is a great advantage compared to reading a thermoluminescent dosimeter (TLD) that needs a certain amount of time after irradiation to its analyze. The objective of this work is the characterization and validation of the semiconductor diode (T600010L PTW) for use in vivo, and real-time, measurements of the dose released in a whole body irradiation treatment. The dosimeters shall be characterized and calibrated for use in the art in question. Thus, the reproducibility, linearity, and dependence of the diode response with the dose rate, the source-detector distance (SSD) and the angle of incidence of the radiation beam were studied. The calibration of five semiconductor diodes (T600010L PTW) was performed by intercomparing with a Farmer ionization chamber and its verification was done with the simulation of a treatment in an anthropomorphic phantom RANDO . Once the dosimeter was adequate and with variations less than $1 \%$, action limits were set for in vivo dosimetry according to the region to be treated and considering factors of uncertainties that are not intrinsic to the detector. We found the maximum limit of $9.6 \%$ in the knee region.

Keywords: in vivo dosimetry; dosimeters semiconductor; diodes; radiotherapy; TBI.

\section{Introdução}

A irradiação de corpo inteiro (TBI, total body irradiation) com feixes de fótons é comumente empregada como parte do regime de condicionamento para transplante de medula óssea. Esse transplante é utilizado no tratamento de uma variedade de doenças tais como leucemia, anemia aplástica, linfoma, mieloma múltiplo, doenças autoimunes, erros inatos do metabolismo, e assim por diante. O papel do TBI é destruir as células tumorais e a medula óssea do receptor, além de causar imunossupressão a fim de evitar a rejeição da medula óssea vinda do doador ${ }^{1}$.

Como é de interesse irradiar o corpo todo, a técnica exige o uso de campos alargados e a consideração de fatores como energia, distância de tratamento, posicionamento do paciente e taxa de dose é determinante para garantir a entrega da dose 
prescrita e a homogeneidade na distribuição de dose, já que se trata de superfície com alta irregularidade. Sendo assim, o TBI é considerado uma técnica de alta complexidade em radioterapia.

Os esquemas de cálculo para determinar, de forma exata, a distribuição da dose no TBI são complexos. Além disso, durante a aplicação, as alterações na posição do paciente, em relação àquela definida no planejamento, mudam dramaticamente as distribuições de dose. Por esta razão é desejável ter uma técnica de medição de dose in vivo disponível. ${ }^{2}$

A dosimetria in vivo monitora diretamente a dose administrada ao paciente durante o tratamento radioterápico, ao contrário de outros métodos de controle de qualidade (QA) realizados antes do tratamento. As medidas in vivo são uma segurança adicional contra possíveis erros que não tenham sido percebidos no decorrer do pré-tratamento. $\mathrm{Na}$ ausência de erros, o registro das medições de rotina, in vivo, é um documento comprobatório de que o tratamento foi entregue corretamente dentro da tolerância especificada. ${ }^{3}$

As câmaras de ionização apresentam dificuldades nesses procedimentos por serem volumosas e frágeis, tornando-as pouco utilizadas na dosimetria in vivo. Além disso, requerem conexão a um eletrômetro e, consequentemente, a aplicação de uma alta voltagem, que pode representar um risco de choque elétrico para o paciente. Dessa forma, para o caso específico de tratamento TBI, essas câmaras possuem aplicação limitada. ${ }^{2}$

Os dosímetros in vivo podem ser classificados quanto ao tempo que transcorre entre irradiação e leitura em dosímetros de tempo real e os de tempo passivo, que precisam de certo intervalo de tempo após a irradiação para sua análise. Ambos os tipos requerem uma calibração que é obtida comparando sua resposta, a uma dose conhecida, com a de uma câmara de ionização calibrada $(\mathrm{Cl}){ }^{4}$

Os diodos semicondutores, quando conectados a um eletrômetro adequado, oferecem uma combinação de alta sensibilidade, leitura imediata, simplicidade de operação (sem tensão de polarização externa), tamanho pequeno e robustez. ${ }^{5}$ Os eletrômetros utilizados neste tipo de procedimento fornecem uma leitura imediata em vários canais simultaneamente. As características e os aspectos práticos do uso dos diodos na dosimetria in vivo estão descritos em grande número de publicações, em várias das quais também se discute a técnica empregada. ${ }^{3,5,6}$

As condições da irradiação de corpo inteiro são diferentes de um tratamento de radioterapia convencional, portanto a calibração do dosímetro deve ser realizada no próprio setup do TBI. A dose apresentada depende de condições geométricas e intrínsecas do detector. Sendo assim, previamente, devem-se avaliar fatores como linearidade, reprodutibilidade e dependência tanto angular como com a taxa de dose.

A corrente de radiação gerada em um diodo pode aumentar ou diminuir com aumento da temperatura, resultando em uma variação de sua sensibilidade que é caracterizada pelo parâmetro SVWT (Sensitivity Variation With Temperature). Devido ao longo tempo de contato do diodo com o paciente no TBI, deve-se conhecer seu SVWT, que é um fator que estabelece a variação percentual da sensibilidade pela variação da temperatura, em graus Celsius. É interessante que esse fator seja pequeno para minimizar o impacto da correção de temperatura, quando essa correção não é aplicada. ${ }^{3}$

A diferença entre a dose medida e a calculada na dosimetria in vivo deve ser inferior a $\pm 5 \%$ como recomenda a International Commission of Radiation Units and Measurements (ICRU). ${ }^{7}$ Porém, devem-se realizar estudos a fim de definir limites de ação, que são parâmetros que protegem contra incertezas associadas à administração e à medida da dose para a técnica, pois garantem que os valores das medidas estarão dentro de um intervalo de confiança, que é o grau de incerteza associado com uma estimativa. Para esta avaliação, devem-se utilizar detectores posicionados nos vários pontos do corpo em que foi realizado o cálculo da dose a ser entregue.

O usuário deve verificar o fator de calibração a cada dois ou três meses (ou a após 5 a 10 pacientes), sendo aceitável uma variação de $2 \%{ }^{3}$ Caso a variação encontrada for superior, deve-se adquirir um novo fator de calibração.

O objetivo deste trabalho é a caracterização do diodo semicondutor (T600010L) para a medida in vivo em tempo real, que permite uma investigação imediata para tratamentos de irradiação de corpo todo.

\section{Materiais e Métodos}

Utilizou-se neste estudo o seguinte conjunto de dispositivos:

a) cinco diodos In-Vivo semi-condutor T600010MP amarelos (Figura 1);

b) eletrômetro VIVODOS T10018;

c) adaptador de Calibração "S" (Figura 2);

d) phantom antropomórfico RANDO®;

e) placas de água sólida;

f) câmara de ionização TW1003 (com capa de build-up para $6 \mathrm{MV}$ );

g) eletrômetro UNIDOS;

h) termômetro;

i) barômetro.

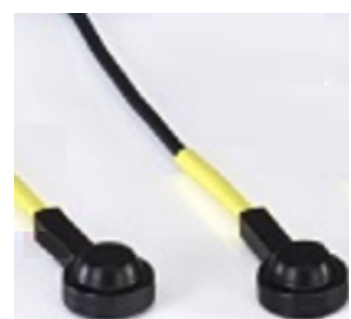

Figura 1. Diodo semicondutor T60010MP amarelo

As especificações técnicas fornecidas pelo fabricante do diodo semicondutor T60010MP são:

- aplicação: medida de dose de entrada e saída;

- energia: fótons de 3 a $15 \mathrm{MV}$;

- material do build-up: chumbo; 
- build-up total: $2,0 \mathrm{~g} / \mathrm{cm}^{2}$

- resposta: $10 \mathrm{nC/Gy}$

- SVWT (variação da sensibilidade com a temperatura): $\leq 0,4 \% / K$;

- estabilidade de dose: $\leq 0,5 \% / \mathrm{kGy}(6 \mathrm{MV}) \mathrm{e}$

- dependência direcional: < $5 \%$ (6 MV).

Com o objetivo de evitar erros grosseiros de posicionamento durante a realização dos testes descritos abaixo, os cinco dosímetros ficaram dispostos em um aparato experimental de acrílico mostrado na Figura 2.
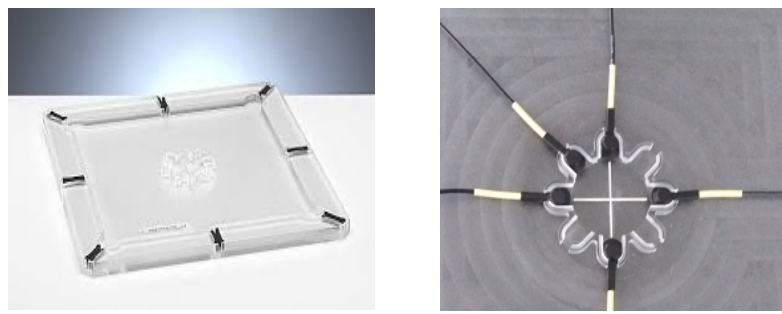

Figura 2. Adaptador de calibração "S"

Os testes realizados em cinco diodos e o respectivo setup foram:

a. linearidade: variando a unidade monitora (UM) em passo de 50 UM, entre 50 e 1000 UM, taxa de dose fixa em $100 \mathrm{UM} / \mathrm{min}$. Foram realizadas 5 medidas em cada diodo.

b. reprodutibilidade: 5 exposições iguais em cada diodo com 500 UM e taxa de dose de 100 $\mathrm{UM} / \mathrm{min}$ realizadas em dias diferentes;

c. dependência com a taxa de dose: 5 exposições em cada diodo, variando a taxa de dose de 100 a $600 \mathrm{UM} / \mathrm{min}$ (valores disponíveis no equipamento) com 500 UM fixas.

d. dependência angular: esta foi avaliada nas condições de referência $(S S D=100 \mathrm{~cm}, 100 \mathrm{UM}$, taxa de dose de $400 \mathrm{UM} / \mathrm{min}$ ) e apenas para dois dos diodos semicondutores (T600010L PTW) variando os ângulos do gantry de $10 \mathrm{em}$ $10^{\circ}$ entre 0 e $90^{\circ}$ e entre 270 e $250^{\circ}$ e nos ângulos de $180^{\circ}$ (posterior do detector) e $235^{\circ}$;

e. dependência com a SSD: com 500 UM e taxa de dose de $100 \mathrm{UM} / \mathrm{min}$, verificou-se a validade da correção pelo inverso do quadrado da distância (IQD) em relação às doses encontradas na distância de referência do TBI $(320 \mathrm{~cm})$ para duas distâncias da fonte à superfície do detector, 330 e $340 \mathrm{~cm}$.

Os diodos foram calibrados utilizando a câmara de ionização como referência para dose absoluta de acordo com a Equação 1:

$$
F_{\text {cal }}=\frac{D_{I C}}{L_{\text {diodo }}}
$$

Sendo $D_{I C}$ a dose na câmara de ionização e $L_{\text {diodo }}$ a leitura no diodo.

A calibração foi realizada no setup do TBI: $S S D=320 \mathrm{~cm}$, campo de $(40 \times 40) \mathrm{cm}^{2}$, gantry a $270^{\circ}$, colimador a $45^{\circ}$, taxa de dose de $100 \mathrm{UM} / \mathrm{min}$. O phantom tinha, na parte central, placas de água sólida como pode ser visto na Figura 3.

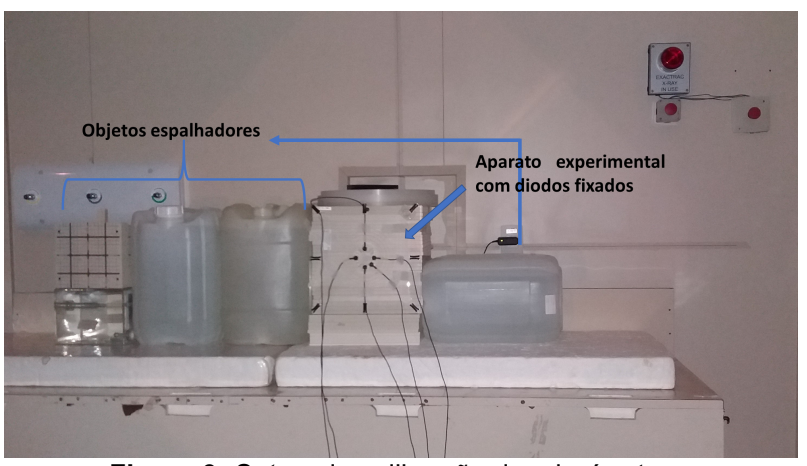

Figura 3. Set up de calibração dos dosímetros

Todas as etapas descritas anteriormente foram feitas com fótons de $6 \mathrm{MV}$, uma vez que é a única utilizada para esta técnica no serviço.

Após a caracterização e calibração do sistema com a finalidade de verificar os fatores obtidos, realizou-se a simulação de um tratamento de TBI, com 200 cGy prescrito no raio central, e respectiva verificação de dose in vivo em um phantom antropomórfico RANDO ${ }^{\circ}$. O resultado foi analisado com a equação (2), que fornece a diferença percentual entre a dose medida e a esperada:

$$
\Delta=\frac{(\text { Dose medida-Dose esperada }) \times 100}{\text { Dose esperada }}
$$

Posteriormente, realizaram-se medidas in vivo no primeiro dia dos tratamentos de TBI em 11 pacientes. Os pontos em que a dose foi avaliada foram cabeça, pescoço, fúrcula, tórax, raio central, joelho e pés. Foram escolhidos sempre cinco pontos por paciente por causa da limitação no número de diodos. O ponto em que se prescreve a dose de tratamento, o raio central, foi medido em todos os pacientes. Novamente o resultado foi analisado com a equação (2), que compara dados medidos com os dados esperados do planejamento.

\section{Resultados}

\subsection{Linearidade}

Os dosímetros mostraram comportamento linear na faixa de dose medida. O gráfico com os dados obtidos apresentou um coeficiente de determinação $\mathrm{R}^{2}$ igual a 1 e intersecções com o eixo $\mathrm{x}$ próximas de zero, como mostra o gráfico da Figura 4.

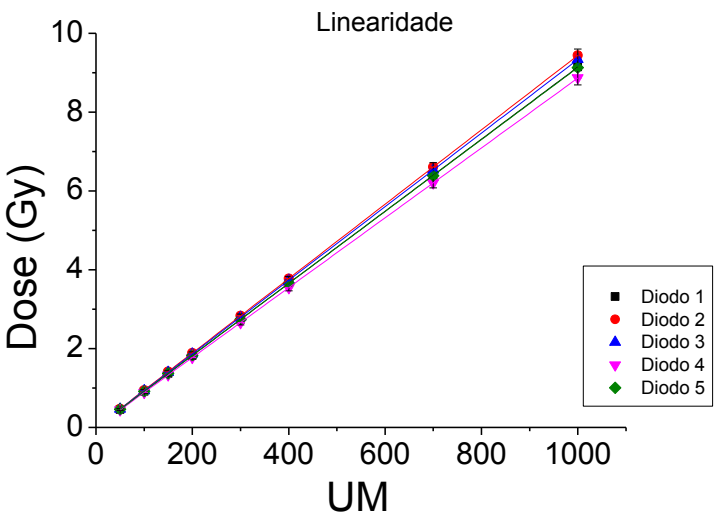

Figura 4. Dose versus UM para os diversos diodos 


\subsection{Reprodutibilidade}

Confirmou-se a reprodutibilidade com um desvio médio de 0,0005 Gy entre as medidas e uma variação da média pelo desvio padrão de $0,1 \%$, representada no gráfico da Figura 5.

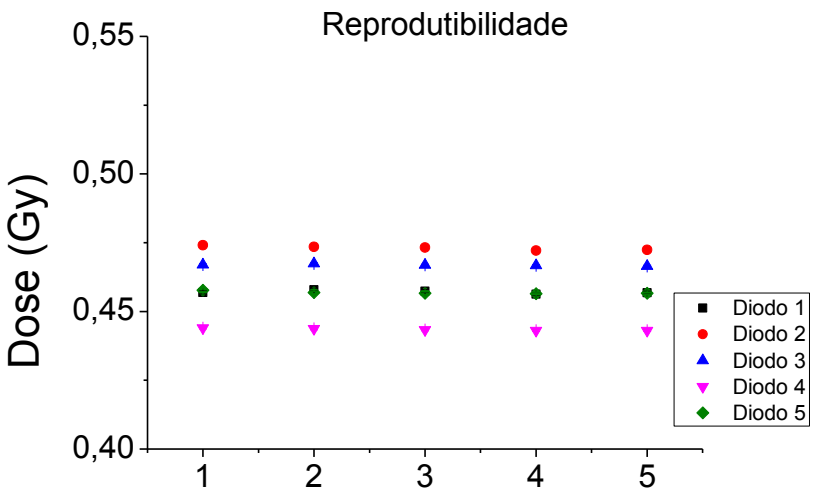

Figura 5. Média das doses (Gy) obtidas em cinco dias aleatórios

\subsection{Dependência com a taxa de dose}

Para essa dependência, o desvio médio encontrado foi de 0,0028 e a variação de $0,6 \%$, conforme mostra o gráfico da Figura 6 .

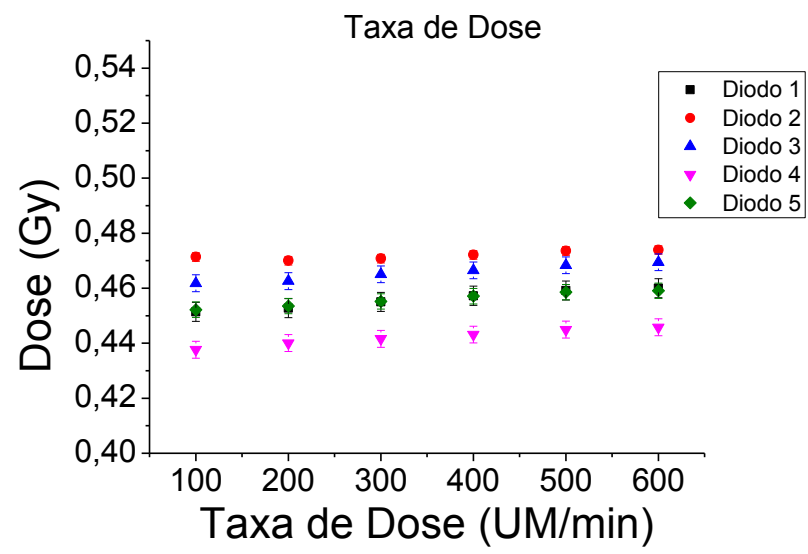

Figura 6. Dose (Gy) versus taxa de dose (UM/min)

\subsection{Dependência angular}

Os dosímetros mostraram alta dependência angular com uma variação de $5,2 \%$ em relação a dose no ângulo zero, conforme apresentado na Figura 7. As doses foram normalizadas para a medida no ângulo de zero graus e seus respectivos valores estão apresentados no eixo vertical do gráfico.

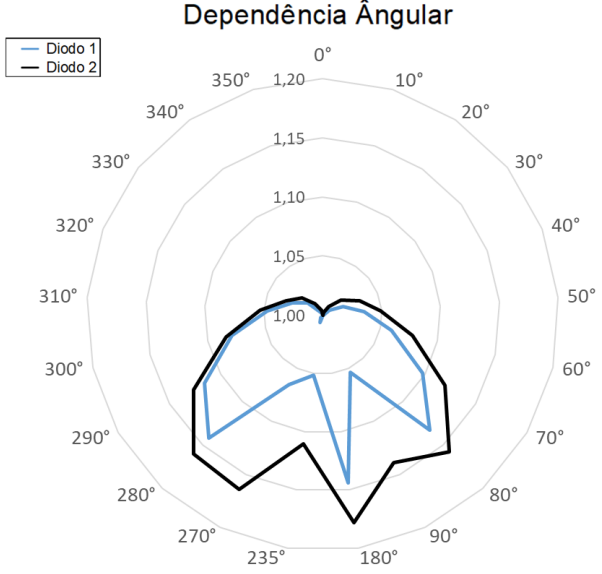

Figura 7. Dose (Gy) normalizada para o ângulo de zero graus versus ângulo do gantry $\left({ }^{\circ}\right)$

\subsection{Verificação da lei do IQD}

Essa verificação foi realizada corrigindo a leitura encontrada na SSD de referência do TBI $(320 \mathrm{~cm})$ pela lei do inverso do quadrado da distância e comparando com os valores obtidos na nova distância. Foi encontrado um desvio máximo de $0,84 \%$ e $-0,34 \%$, respectivamente, para as SSD de $330 \mathrm{~cm}$ e $340 \mathrm{~cm}$, Tabela 1.

\begin{tabular}{|c|c|c|c|c|}
\hline \multirow[b]{3}{*}{ Diodo } & \multicolumn{4}{|c|}{ Dose } \\
\hline & \multirow{2}{*}{$\begin{array}{c}\text { SSD } 320 \\
\text { Referência (Gy) }\end{array}$} & \multicolumn{3}{|c|}{ SSD 330} \\
\hline & & Esperada teórica (Gy) & Medida (Gy) & Desvio $(\%)$ \\
\hline 1 & 0,457 & 0,430 & 0,427 & 0,769 \\
\hline 2 & 0,473 & 0,445 & 0,441 & 0,844 \\
\hline 3 & 0,467 & 0,439 & 0,436 & 0,788 \\
\hline 4 & 0,443 & 0,417 & 0,414 & 0,669 \\
\hline \multirow[t]{2}{*}{5} & 0,457 & 0,430 & 0,426 & 0,796 \\
\hline & SSD 320 & \multicolumn{3}{|c|}{ SSD 340} \\
\hline Diodo & Referência (Gy) & Esperada teórica (Gy) & Medida (Gy) & Desvio $(\%)$ \\
\hline 1 & 0,457 & 0,405 & 0,406 & $-0,327$ \\
\hline 2 & 0,473 & 0,419 & 0,419 & $-0,085$ \\
\hline 3 & 0,467 & 0,414 & 0,415 & $-0,341$ \\
\hline 4 & 0,443 & 0,393 & 0,393 & $-0,126$ \\
\hline 5 & 0,457 & 0,405 & 0,405 & $-0,125$ \\
\hline
\end{tabular}

Os fatores de calibração para cada dosímetro foram obtidos por intercomparação com uma câmara de ionização. Como os diodos apresentam uma queda de sensibilidade com o tempo, foram reavaliados após 10 meses, quando apresentaram um desvio médio de aproximadamente $2 \%$ e máximo de $2,6 \%$.

Usando o fator de calibração, realizou-se a medida de dose no phantom antropomórfico. A Tabela 2 mostra a comparação entre as medidas feitas em tempo real e aquelas calculadas. 
Tabela 2. Dose calculada no phantom antropomórfico e dose medida com os diodos

\begin{tabular}{|c|c|c|c|}
\hline Região & Dose calculada (Gy) & Dose medida (Gy) & Desvio (\%) \\
\hline cabeça & 0,742 & 0,784 & 5,53 \\
fúrcula & 0,737 & 0,766 & 3,83 \\
\hline mediastino & 0,754 & 0,772 & 2,36 \\
\hline abdome (RC) & 0,736 & 0,750 & 1,89 \\
\hline joelho & 0,811 & 0,808 & $-0,324$ \\
\hline
\end{tabular}

A Tabela 3 apresenta a diferença entre os valores medidos e calculados, de acordo com a equação (2), para cada ponto.

Tabela 3. Resultados da soma das medidas de dose de entrada feitas com diodos semicondutores (T600010L PTW) nos campos anterior e posterior somados, in vivo, de pacientes que realizaram

TBI. $n$ é o número de pacientes avaliados em cada ponto.

\begin{tabular}{|l|c|c|c|}
\hline Região & $\mathrm{n}$ & Diferença Média (\%) & Desvio Padrão da Diferença \\
\hline Cabeça & 10 & 4,66 & 3,19 \\
\hline Furcula ou Pescoço & 6 & 3,73 & 4,03 \\
\hline Tórax & 7 & 2,29 & 4,83 \\
Raio Central & 11 & 1,80 & 5,68 \\
\hline Joelho & 6 & 3,34 & 5,74 \\
\hline Pés & 8 & $-1,44$ & 3,76 \\
\hline
\end{tabular}

Em todos os tratamentos também foram realizadas medidas com dosímetro termoluminescente (TLD) nas mesmas regiões, conforme apresentado na Tabela 4. Na nossa instituição, o limite de ação para a diferença entre o medido e o calculado para a dosimetria TLD in vivo é de $\pm 10 \%$ para o TBI. Como é trabalhoso fazer uma seleção de TLDs com resposta numa faixa estreita de valores, a seleção feita no serviço inclui dosímetros com respostas, a uma mesma dose, com variações médias de $\pm 5 \%$. Somando-se a esse valor a incerteza nas medidas, considera-se aceitável uma tolerância de $10 \%^{8}$. Os diodos apresentaram valores inferiores a esse limite.

Tabela 4. Resultados da soma das medidas de dose de entrada feitas com TLD nos campos anterior e posterior de pacientes que realizaram TBI.

\begin{tabular}{|l|c|c|}
\hline Região & Diferença Média (\%) & Desvio Padrão da Diferença \\
\hline Cabeça & 0,932 & 5,50 \\
\cline { 2 - 3 } Tórax & 0,339 & 5,79 \\
\cline { 2 - 3 } Raio Central & 3,23 & 5,56 \\
\cline { 2 - 3 } Joelho & 0,865 & 6,29 \\
\hline Pés & $-3,25$ & 6,82 \\
\hline
\end{tabular}

\section{Discussão}

\subsection{Caracterização}

O diodo semicondutor (T600010L PTW) usado como dosímetro mostrou-se linear na faixa de dose medida, com a reprodutibilidade de $0,1 \%$ e uma dependência média com taxa de dose de $0,6 \%$. 0 Report $87^{3}$ recomenda que as variações desses parâmetros para este dosímetro sejam menores que $1 \%$. Desta forma, o diodo usado é ideal para medidas in vivo no TBI.

As análises mostraram uma alta dependência angular já que este tipo de diodo possui uma capa de build up plana embutida, conforme desenho representativo da Figura 8 , que desfavorece a incidência não perpendicular. Seria inviável achar um fator de correção para esta dependência, visto que na dosimetria in vivo não seria possível determinar o ângulo exato em que o diodo estaria preso no paciente. A solução encontrada foi evitar incidências oblíquas.

\section{$1 \mathrm{~mm}^{2}$ de região ativa \\ Build-up embutido \\ Figura 8. Desenho representativo do diodo semicondutor T60010MP amarelo}

Report 17 da $A A P M^{2}$ sugere que diferenças maiores de $2 \%$ no cálculo do inverso do quadrado da distância geram a necessidade de utilização de um fator de calibração para um número apropriado de distâncias utilizadas na rotina. Os resultados obtidos dispensam a necessidade de um fator de correção para outras distâncias, visto que o maior desvio encontrado foi de $0,84 \%$ para SSD de 330 $\mathrm{cm}$.

\subsection{Calibração}

A verificação do fator de calibração utilizado foi realizada com a simulação de tratamento no phantom antropomórfico, conforme figura 9 abaixo.

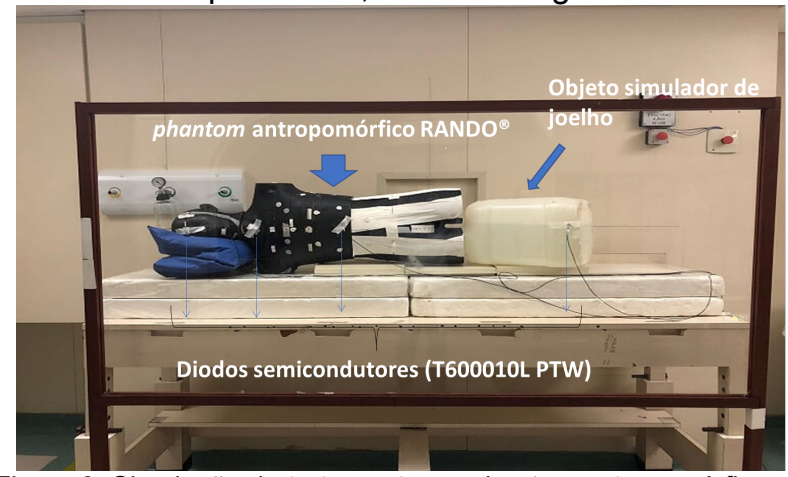

Figura 9. Simulação de tratamento no phantom antropomórfico os diodos semicondutores (T600010L PTW) posicionados

Os desvios entre a dose calculada e a medida foram satisfatórios, já que são menores de $5 \%$, como recomenda o ICRU 50 ${ }^{7}$, com exceção da cabeça (tabela 2) devido ao posicionamento angulado já discutido anteriormente, o que mostra que o fator de calibração estava correto.

O Report $87^{3}$ recomenda uma recalibração dos dosímetros quando seus fatores de calibração atingem o valor de $2 \%$ de variação. Considerando o número de pacientes tratados no serviço, e que os valores encontrados foram próximos dessa tolerância, as verificações serão realizadas bimestralmente garantindo que não haja erro introduzido pelo fator de calibração.

\subsection{Dosimetria in vivo}

As medidas com TLD apresentaram, de uma forma geral, valores médios de desvios entre os 
medidos e os planejados inferiores aos dos diodos, porém com desvio padrão maior.

Uma vantagem da utilização do diodo em relação ao TLD é a leitura imediata, sendo que, a leitura dos TLDs na instituição é realizada com, no mínimo, 6 horas após a irradiação, além de exigir um tratamento térmico antes de ser utilizado. Este intervalo de horas para a leitura é necessário para evitar os efeitos da supralinearidade, fenômeno que deve ser considerado para as doses utilizadas em radioterapia, e para evitar possíveis ruídos causados por elétrons que estão na banda de valência ${ }^{8}$. O uso do TLD não permite a detecção imediata de um erro na irradiação.

As medidas realizadas nos onze pacientes possibilitaram o estabelecimento de um limite de ação para o dosímetro, que levasse em consideração fatores como posicionamento do detector, possíveis movimentações do paciente ou imprecisão no cálculo manual de compensadores para determinada região.

Para definir o limite de ação, considerando uma população com $n<30$, usou-se a tabela de distribuição t-student com um nível de confiança da média de $95 \%$. O cálculo do limite foi feito através da equação (3), onde $\alpha=0,05, \Delta$ é a diferença média entre o limite calculado e o medido (\%) e n é a população, com os valores apresentados na Tabela 3. Os dados obtidos estão apresentados na Tabela 5.

$$
\mu=\Delta_{\text {medio }} \pm t_{\frac{\alpha}{2}, n-1} \frac{\text { Desvio Padrão }}{\sqrt{N}}
$$

Tabela 5. Limites de ação encontrados com base nos resultados apresentados na Tabela 3

\begin{tabular}{|l|c|}
\hline Região & Limite de ação (\%) \\
\hline Cabeça & 6,94 \\
\hline Fúrcula ou Pescoço & 7,96 \\
\hline Tórax & 6,76 \\
\hline Raio Central & 5,62 \\
\hline Joelho & 9,37 \\
\hline Pés & 4,58 \\
\hline
\end{tabular}

Os limites de ação encontrados, em sua maioria, foram maiores do que a recomendação do ICRU 50, de $5 \%$. Porém, muitos fatores existentes na prática clínica contribuem para um valor maior de discrepância entre o calculado e o medido. Entre eles pode-se citar:

- as posições dos pontos em que a dose foi calculada e medida podem não ser idênticas, já que os dias de planejamento e tratamento são distintos;

- a movimentação do paciente durante o tratamento pode ser grande, pois essa é uma técnica que demanda tempo;

- pode ocorrer uma pequena diferença na distância de posicionamento do paciente;

- as medidas são realizadas na superfície do paciente onde há imprecisão na entrega de dose, apesar de se usar uma capa de build-up fixa no dosímetro;

- em algumas superfícies encontra-se dificuldade em se manter o dosímetro perpendicular ao feixe e

- pode ocorrer a não estabilização da temperatura do dosímetro.

Não há necessidade de fazer a correção do inverso do quadrado da distância para a SSD utilizada, uma vez que encontramos variações menores que $2 \%$. Porém, para diminuir as incertezas, essa seria uma solução interessante.

Diversos artigos, devido a essas incertezas, estabeleceram como limites de ação valores superiores a $5 \% \%^{9,10,11,12}$.

A variação na sensibilidade do detector de acordo com a temperatura fornecida pelo fabricante é de $0,4 \% / \mathrm{K}\left(0,4 \% /{ }^{\circ} \mathrm{C}\right)^{13}$ e pode ser reduzida com a utilização de uma capa isolante entre o dosímetro e a pele do paciente. O desenvolvimento de uma capa de isopor está sendo avaliada.

E apesar de, nas nossas avaliações, os limites de ação para a diferença entre os dados medidos e planejados estarem acima de $5 \%$ em alguns pontos, a homogeneidade de dose entre os diferentes pontos do corpo e o ponto de cálculo (raio central) está dentro do recomendado pelo protocolo (AAPM, $1986)$, ou seja, $\pm 10 \%$.

\section{Conclusões}

Conclui-se que o diodo semicondutor (T600010L PTW) possui desempenho adequado para utilização na dosimetria in vivo na técnica de irradiação de corpo inteiro, pois a variação apresentada nas leituras durante a caracterização é menor que $1 \%$.

A utilização do diodo semicondutor (T600010L PTW) como dosímetro em incidências inclinadas não é recomendada, devido à dependência angular existente, resultado da geometria e características técnicas do dosímetro, como por exemplo, sua capa de build-up embutida.

A verificação na queda de sensibilidade diodo semicondutor (T600010L PTW) realizada após dez meses não foi concordante com os limites de $2 \%$ estabelecidos pelo Report $87^{3}$. Então, foi estabelecida uma periodicidade de dois meses para a calibração dos diodos.

As medidas in vivo obtidas foram adequadas e, através delas, foi estabelecido um limite de ação por região que assume, além das incertezas do diodo semicondutor (T600010L PTW), as da técnica de TBI e as do posicionamento, tanto do paciente quanto do dosímetro. O limite máximo encontrado foi de $9,6 \%$ no joelho. Com o aumento do número de pacientes será possível melhorar a estatística e estabelecer um limite de ação mais confiável.

\section{Agradecimentos}

Ao apoio financeiro da SGTES-Ministério da Saúde e da SESu-Ministério da Educação e a toda a equipe de Radioterapia do HCFMUSP pela colaboração com o trabalho, em especial a Laura Furnari e Caroline Zepellini pela orientação e paciência e aos físicos Gabriela Reis e Marcos Vinicius Nakandakari as discussões construtivas. 


\section{Referências}

1. Khan, Faiz M. The Physics of Radiation Therapy, 3rd Edition, CAP 18, pg 456.

2. AAPM (American Association of Physicists in Medicine). REPORT 17 - The Physical Aspects of Total and Half Body Photon Irradiation, 1986.

3. AAPM (American Association of Physicists in Medicine). REPORT 87 - Diode in vivo Dosimetry for Patients Receiving External Beam Radiation Therapy, 2005.

4. Ben Mijnheera, Sam Beddar, Joanna Izewska, Chester Reft In vivo dosimetry in external beam radiotherapy, Medical Physics, 2013.

5. IAEA (International Atomic Energy Agency). REPORT 8 Development of procedures for in vivo Dosimetry in Radiotherapy, 2013.

6. ESTRO. Booklet 5 - Practical Guidelines for the Implementation of in vivo Dosimetry with diodes in external radiotherapy with Photon beams (entrance dose), 2001

7. International Commission on Radiation Units and Measurements (ICRU). Report 50 - Prescribing, Recording and Reporting Photon Beam Therapy ICRU, 1993.

8. Habitzreuter, Ângela Beatriz ; Rodrigues, Laura Natal. Implementação da irradiação de corpo inteiro em radioterapia, 2010.

9. Lanchun Lu et al. A Clinical Dosimetry Analysis of Total Body Irradiation for Leukemia Patients, Medical Physics, 2014.

10. Lancaster, C. M.; Crosbie, J. C. and Davis, S. R. In-vivo dosimetry from total body irradiation patients $(2000-2006)$ : results and analysis, Australasian Physical \& Engineering Sciences in Medicine, 2008.

11. Dupont, S.; Aubignac, L.; Dufreneix, S.; Briand, C.; Jaffre, F.; Klotz, S. et al. Contrôle qualité de la dose délivrée par dosimétrie in vivo : un critère de tolérance unique peut-il satisfaire toutes les localisations?, Cancer/Radiothérapie, 2012.

12. Rittmann, K. L. Quality assurance in Total Body Irradiation by in vivo dosimentry, 1996.

13. Application-Specific In Vivo Semiconductor Diodes for VIVODOS and MULTIDOS ${ }^{\circ}$ Dosemeters (http://www.ptw.de/2337.html?\&cld=3272).

\section{Contato:}

Caroline Castilhano Sampaio

E-mail: caroline.castilhano@hc.fm.usp.br 